Allergology today

\section{Versteckte Allergene in Alkohol-Mixgetränken}

Beim Genuss von alkoholischen Getränken können auch versteckte Allergene zum Verhängnis werden. So kam es bei einer 19-jährigen Frau mehrmals nach Konsum von alkoholhaltigen Mixgetränken zu anaphylaktischen Reaktionen, die von Kribbeln an den Händen bis zu Urtikaria, Quincke-Ödem, Übelkeit und Atemnot reichten. Pricktests mit verschiedenen Alkoholika sowie orale Provokationstestungen blieben negativ. Auf Bitter
Lemon und Tonic Water dagegen kam es zu positiven Reaktionen. Mittels Pricktestung konnte schließlich eine Typ-1-Allergie aufChininsulfat diagnostiziert werden, berichtete Jarmila Liptak von der TU München.

Chinin wird nicht nur als Malariamittel und Muskelrelaxans eingesetzt, sondern auch als bitter schmeckendes Aroma verschiedenen Getränken zugesetzt. Da dieser Inhaltsstoff nur in alkoholfreien Getränken deklarations-

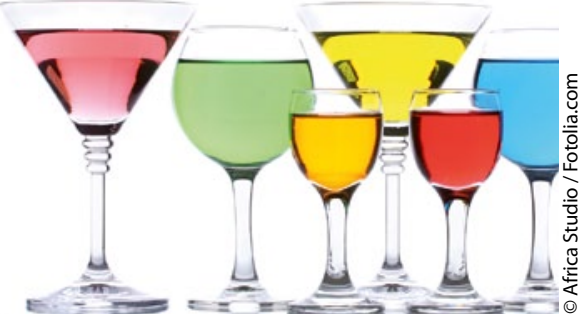

pflichtig ist, müssen betroffene Allergiker vorsichtig sein. Angelika Bauer-Delto

Liptak J, Kigitsidou E, Ring J, Brockow K, Darsow U. Anaphylaktische Reaktionen nach Genuss von Alkohol-Mixgetränken

\section{Mikroben verstärken allergische Immunantwort}

Die Immunantwort auf Allergene wird durch eine Vielzahl von Faktoren beeinflusst. Eine Arbeitsgruppe um Bärbel Heydenreich von der Universitäts-Hautklinik Mainz ging in Zusammenarbeit mit dem Forschungszentrum Borstel der Frage nach, ob beispielsweise eine Mikrobenbesiedelung von Pollen adjuvante immunologische Effekte ausübt. Die Forscher sammelten hierzu Lieschgraspollen, säten diese auf Agarplatten aus und kultivierten und bestimmten die wachsenden Mikroorganismen. Es wurde eine komplexe Mischung verschiedener Bakterien und Schimmelpilze auf den Gräserpollen detektiert. Neben Gram-negativen Bakterien, für die eine TH1-fördernde Wirkung bekannt ist, sowie Schimmelpilzen, die selbst als Allergene wirken, wurden in großen Mengen die Gram-positiven Bakterien Bacillus cereus und Bacillus subtilis gefunden.

In vitro führte die Zugabe von Überständen homogenisierter Gram-positiver Bakterien zu einer vermehrten Ausreifung dendritischer Zellen (DC) von Gräserpollen-Allergikern und zu einer erhöhten Freisetzung von IL-6, IL-12p40 sowie TNF-a. Die Stimulation von $\mathrm{CD}^{+}{ }^{+}-\mathrm{T}-$ Zellen mit $\mathrm{DC}$, die mit Allergen und Überständen homogenisierter Bakterien behandelt worden waren, führte im Vergleich zur Stimulation mit nur Aller-

gen-gepulsten DC zu einer verstärkten Proliferation sowie einer vermehrten Produktion von IL-4, IL-13, IL-10, IL-17, IL-22 und IFN- $\gamma$.

Die Besiedelung von Gräserpollen mit Mikroorganismen kann somit allergische Reaktionen verstärken, schlussfolgerte Heydenreich. Gram-positive Bakterien zeigen eine Adjuvanswirkung, indem sie die Ausreifung von DC fördern und TH1-, TH2- und TH17-vermittelte allergische Immunantworten induzieren.

Angelika Bauer-Delto

Heydenreich B, Bellinghausen I, Becker W, Grabbe S, Petersen A, Saloga J. Adjuvanswirkung von Gram-positiven Bakterien auf Gräserpollen

\section{„Heuschnupfen" durch Heuschrecke}

Rhinokonjunktivale Symptome können auch durch Insekten verursacht werden. Das zeigt der Fall einer Patientin, den Dr. Ulrich May von der Klinik und Poliklinik für Dermatologie und Allergologie der TU München vorstellte: Bei der 30-jährigen Patientin waren erstmals vor sechs Monaten an ihrem Arbeitsplatz rhinokonjunktivale Beschwerden aufgetreten. Die
Patientin litt seit der Kindheit an rezidivierenden Ekzemen, Typ-1-Allergien waren nicht bekannt.

Die Pricktestung mit Standard-Aeroallergenen, die Pollen, Hausstaubmilben, Tierhaare und Schimmelpilze umfasste, blieb durchweg negativ. Das Gesamt-IgE war mit 27,9 IU/ml niedrig. Im Atopene-RAST ergab sich nur für

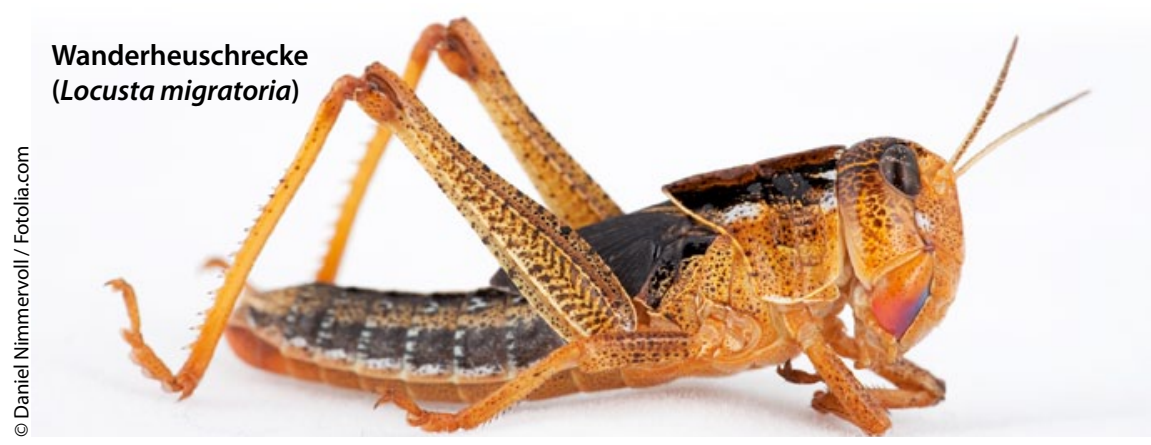

Birkenpollen Klasse 1, die Sensibilisierung war klinisch jedoch asymptomatisch. Da die Patientin im Rahmen ihrer Doktorarbeit seit zwei Jahren mit Wanderheuschrecken arbeitete, wurde schließlich auch eine Pricktestung mit Flügel und Körper der Heuschrecke durchgeführt. Das Ergebnis war positiv. Eine Kreuzreaktivität mit Motte, Mücke, Küchenschabe und Milbe liegt nicht vor.

Diagnostiziert wurde eine Typ 1-Allergie auf Wanderheuschrecken. Die allergischen Beschwerden der Patientin wurden symptomatisch mit Antihistaminika behandelt. Durch Schutzmaßnahmen wie das Tragen von Partikelfiltern kann die Allergenbelastung reduziert werden. Zudem wurde eine Allergenkarenz empfohlen, die allerdings erst nach Abschluss der Doktorarbeit eingehalten wurde.

Angelika Bauer-Delto

May U, Ollert M, Ring J, Darsow U. Typ-I-

Allergie auf Wanderheuschrecke 